\title{
Performance of GARCH models in forecasting stock market volatility.
}

\begin{abstract}
This paper studies the performance of GARCH model and its modifications, using the rate of returns from the daily stock market indices of the Kuala Lumpur Stock Exchange (KLSE) including Composite Index, Tins Index, Plantations Index, Properties Index, and Finance Index. The models are stationary GARCH, unconstrained GARCH, non-negative GARCH, GARCH-M, exponential GARCH and integrated GARCH. The parameters of these models and variance processes are estimated jointly using the maximum likelihood method. The performance of the within-sample estimation is diagnosed using several goodness-of-fit statistics. We observed that, among the models, even though exponential GARCH is not the best model in the goodness-of-fit statistics, it performs best in describing the often-observed skewness in stock market indices and in out-of-sample (one-step-ahead) forecasting. The integrated GARCH, on the other hand, is the poorest model in both respects.
\end{abstract}

Keyword: Forecasting volatility; GARCH; Time-series; Rate of returns. 